\title{
FOURTH-ORDER TWO-POINT BOUNDARY VALUE PROBLEMS
}

\author{
YISONG YANG
}

(Communicated by Kenneth R. Meyer)

\begin{abstract}
We establish the uniqueness and existence theorems for a linear and a nonlinear fourth-order boundary value problem at nonresonance.
\end{abstract}

1. Introduction. Fourth-order two-point boundary value problems are essential in describing a vast class of elastic deflection. Usmani [4] studied the problem

$$
\begin{aligned}
& \left(d^{4} / d x^{4}-f(x)\right) y=g(x), \quad 0<x<1, \\
& y(0)=y_{0}, \quad y(1)=y_{1}, \quad y^{\prime \prime}(0)=\bar{y}_{0}, \quad y^{\prime \prime}(1)=\bar{y}_{1} .
\end{aligned}
$$

He proved a uniqueness and existence theorem under condition $\sup |f(x)|<\pi^{4}$. (In his paper [4], the condition is stated in a weaker form $\sup f(x)<\pi^{4}$, however, his proof is valid only for $\sup |f(x)|<\pi^{4}[\mathbf{5}]$.) Attempts have been made to establish the existence for the following general nonlinear problem

$$
\begin{aligned}
& d^{4} y / d x^{4}=f\left(x, y, y^{\prime \prime}\right), \quad 0<x<1, \\
& y(0)=y_{0}, \quad y(1)=y_{1}, \quad y^{\prime \prime}(0)=\bar{y}_{0}, \quad y^{\prime \prime}(1)=\bar{y}_{1} .
\end{aligned}
$$

For example, Aftabizadeh [1] proved an existence theorem under the severe condition that $f$ is a bounded function. In the present paper we study the above problems under more general conditions.

2. The nonlinear problem. In this section we establish the following general result for problem (2).

THEOREM 1. Suppose that $f(x, y, u)$ is continuous on $[0,1] \times R \times R$ and there are constants $a, b, c \geq 0$ such that

$$
|f(x, y, u)| \leq a|y|+b|u|+c, \quad \text { where } a / \pi^{4}+b / \pi^{2}<1 .
$$

Then for any $y_{0}, y_{1}, \bar{y}_{0}, \bar{y}_{1} \in R$, problem (2) has a solution.

We will give examples to show that condition (3) is sharp.

The proof is carried out using the Leray-Schauder degree theory $[2,3]$, but first a lemma is proved concerning a priori estimates for solutions of the problems

$$
\begin{aligned}
& d^{4} y / d x^{4}=t f\left(x, y, y^{\prime \prime}\right), \quad 0<x<1, \\
& y(0)=t y_{0}, \quad y(1)=t y_{1}, \quad y^{\prime \prime}(0)=t \bar{y}_{0}, \quad y^{\prime \prime}(1)=t \bar{y}_{1} .
\end{aligned}
$$

Received by the editors June 2, 1987 and, in revised form, August 5, 1987. 1980 Mathematics Subject Classification (1985 Revision). Primary 34B05, 34B10.

Key words and phrases. Leray-Schauder degree, Fredholm alternative, nonresonance. 
LEMMA. Assume the hypotheses of Theorem 1. Then there exists a constant $M>0$ such that for any $t \in[0,1]$ and any solution $y_{t}(x)$ to problem $\left(2_{t}\right)$, we have

$$
\left\|y_{t}\right\|_{\infty}+\left\|y_{t}^{\prime \prime}\right\|_{\infty}=\sup _{x}\left|y_{t}(x)\right|+\sup _{x}\left|y_{t}^{\prime \prime}(x)\right| \leq M \text {. }
$$

PrOOF. Choose a smooth function $w(x), x \in[0,1]$, such that

$$
w(0)=y_{0}, \quad w(1)=y_{1}, \quad w^{\prime \prime}(0)=\bar{y}_{0}, \quad w^{\prime \prime}(1)=\bar{y}_{1} .
$$

Consider $y_{t}(x)=z_{t}(x)+t w(x)$. If $y_{t}$ is a solution to $\left(2_{t}\right)$, then $z_{t}$ satisfies

$$
\begin{aligned}
d^{4} z / d x^{4} & =t f\left(x, t w(x)+z, t w^{\prime \prime}(x)+z^{\prime \prime}\right)-t w^{(4)}(x) \\
& =g\left(x, z, z^{\prime \prime}\right), \quad 0<x<1, \\
z(0) & =z(1)=z^{\prime \prime}(0)=z^{\prime \prime}(1)=0 .
\end{aligned}
$$

Here $g$ depends on $t$ and satisfies

$$
|g(x, z, u)| \leq a|z|+b|u|+c_{1}
$$

with $a, b$ satisfying (3) and constant $c_{1}$ depending on $c$ in (3) and $w(x)$.

Set $u=z^{\prime \prime}$. We have the following coupled problems

$$
\begin{aligned}
& z^{\prime \prime}=u, \quad z(0)=z(1)=0, \\
& u^{\prime \prime}=g(x, z, u), \quad u(0)=u(1)=0 .
\end{aligned}
$$

From $\left(5^{\prime}\right)$ we have $z z^{\prime \prime}=z u$, and, by first using the Schwarz inequality and then the Poincare inequality,

$$
\begin{aligned}
\int_{0}^{1}\left(z^{\prime}\right)^{2}= & -\int_{0}^{1} z u \leq\left(\int_{0}^{1} z^{2}\right)^{1 / 2}\left(\int_{0}^{1} u^{2}\right)^{1 / 2} \\
& \leq \frac{1}{\pi}\left(\int_{0}^{1}\left(z^{\prime}\right)^{2}\right)^{1 / 2} \frac{1}{\pi}\left(\int_{0}^{1}\left(u^{\prime}\right)^{2}\right)^{1 / 2} .
\end{aligned}
$$

Consequently

$$
\left(\int_{0}^{1}\left(z^{\prime}\right)^{2}\right)^{1 / 2} \leq \frac{1}{\pi^{2}}\left(\int_{0}^{1}\left(u^{\prime}\right)^{2}\right)^{1 / 2}
$$

From $\left(5^{\prime \prime}\right)$ we have $u u^{\prime \prime}=u g(x, z, u)$, and, by using the Schwarz inequality, inequality $2 A B \leq \varepsilon A^{2}+B^{2} / \varepsilon$, and the condition (6) for $g$, we have

$$
\begin{aligned}
\int_{0}^{1}\left(u^{\prime}\right)^{2} & =-\int_{0}^{1} u g(x, z, u) \leq \int_{0}^{1}\left(a|u z|+b u^{2}+c_{1}|u|\right) \\
& \leq a\left(\int_{0}^{1} u^{2}\right)^{1 / 2}\left(\int_{0}^{1} z^{2}\right)^{1 / 2}+b \int_{0}^{1} u^{2}+\frac{1}{2} \varepsilon \int_{0}^{1} u^{2}+\frac{c_{1}^{2}}{2 \varepsilon} .
\end{aligned}
$$

From the Poincaré inequality and (7) we have

$$
\left(\int_{0}^{1} z^{2}\right)^{1 / 2} \leq \frac{1}{\pi}\left(\int_{0}^{1}\left(z^{\prime}\right)^{2}\right)^{1 / 2} \leq \frac{1}{\pi^{3}}\left(\int_{0}^{1}\left(u^{\prime}\right)^{2}\right)^{1 / 2}
$$

and

$$
\left(\int_{0}^{1} u^{2}\right)^{1 / 2} \leq \frac{1}{\pi}\left(\int_{0}^{1}\left(u^{\prime}\right)^{2}\right)^{1 / 2}
$$


Therefore (8) becomes

$$
\int_{0}^{1}\left(u^{\prime}\right)^{2} \leq \frac{a}{\pi^{4}} \int_{0}^{1}\left(u^{\prime}\right)^{2}+\frac{b}{\pi^{2}} \int_{0}^{1}\left(u^{\prime}\right)^{2}+\frac{\varepsilon}{2 \pi^{2}} \int_{0}^{1}\left(u^{\prime}\right)^{2}+\frac{c_{1}^{2}}{2 \varepsilon} .
$$

Since $a, b$ satisfy (3), we can choose $\varepsilon>0$ sufficiently small such that

$$
1-\left(a / \pi^{4}+b / \pi^{2}+\varepsilon / 2 \pi^{2}\right)=K>0 .
$$

Then it follows from (9) that

$$
\int_{0}^{1}\left(u^{\prime}\right)^{2} \leq \frac{c_{1}^{2}}{2 K \varepsilon}=c_{2}
$$

and consequently from (7) and (10) that

$$
\left(\int_{0}^{1}\left(z^{\prime}\right)^{2}\right)^{1 / 2} \leq \frac{\sqrt{c_{2}}}{\pi^{2}}
$$

In particular, (10) and (11) give us

$$
|u(x)|=\left|\int_{0}^{x} u^{\prime}\right| \leq\left(\int_{0}^{1}\left(u^{\prime}\right)^{2}\right)^{1 / 2} \leq \sqrt{c_{2}}
$$

and

$$
|z(x)|=\left|\int_{0}^{x} z^{\prime}\right| \leq\left(\int_{0}^{1}\left(z^{\prime}\right)^{2}\right)^{1 / 2} \leq \frac{\sqrt{c_{2}}}{\pi^{2}} .
$$

Then estimate (4) follows from estimates (12) and (13).

PROOF OF THEOREM. Let $G(x, s)$ be the Green's function of problem

$$
v^{\prime \prime}(x)=h(x), \quad 0<x<1, \quad v(0)=v(1)=0 .
$$

Then

$$
v(x)=\int_{0}^{1} G(x, s) h(s) d s .
$$

In problem $\left(2_{t}\right)$, let $u(x)=y^{\prime \prime}(x)$. Then $\left(2_{t}\right)$ is equivalent to the following system of integral equations

$$
\begin{gathered}
u(x)=t \bar{y}_{0}+x t\left(\bar{y}_{1}-\bar{y}_{0}\right)+\int_{0}^{1} t G(x, s) f(s, y(s), u(s)) d s \\
y(x)=t y_{0}+x t\left(y_{1}-y_{0}\right)+\int_{0}^{1} G(x, s) u(s) d s .
\end{gathered}
$$

Define a function space $X=C[0,1] \times C[0,1]$ with norm $\|(u, y)\|=\|u\|_{\infty}+\|y\|_{\infty}$ for $(u, y) \in X$. Then $X$ is a Banach space. Define a map $T_{t}: X \rightarrow X$ by $T_{t}(u, y)=$ $\left(T_{t}^{\prime}(u, y), T_{t}^{\prime \prime}(u, y)\right)$ where $T_{t}^{\prime}(u, y)$ and $T_{t}^{\prime \prime}(u, y)$ are defined by the right-hand sides of $\left(14^{\prime}\right)$ and $\left(14^{\prime \prime}\right)$ respectively. To prove that problem (2) has a $C^{4}$-solution, we have only to show that $T_{1}$ has a fixed point in $X$. Using the Arzela-Ascoli lemma we easily see that $\left\{T_{t}: t \in[0,1]\right\}$ is a compact operator family from $X$ to $X$. Consider a ball $B_{M}$ in space $X$ :

$$
B_{M}=\{(u, y) \in X:\|(u, y)\| \leq M+1\} .
$$


Estimate (4) says that $T_{t}$ has no fixed point on $\partial B_{M}$. Let Id: $X \rightarrow X$ be the identity map, then Id- $T_{t}$ has no zero on $\partial B_{M}$. Thus by the homotopy invariance of the Leray-Schauder degree, we have by using $T_{0}=0$ that

$\operatorname{deg}\left(\operatorname{Id}-T_{1}, B_{M}, 0\right)=\operatorname{deg}\left(\operatorname{Id}-T_{t}, B_{M}, 0\right)=\operatorname{deg}\left(\operatorname{Id}-T_{0}, B_{M}, 0\right)=\operatorname{deg}\left(\operatorname{Id}, B_{M}, 0\right)=1$.

Consequently, $T_{1}$ has a fixed point in $B_{M}$. The theorem is proved.

Note. A uniqueness theorem can also be obtained if we assume that $f$ satisfies a Lipschitz condition in $y$ and $u$ with constants $a, b$ satisfying (3). The argument is similar to the proof of the above lemma.

EXAMPLES. If $y_{0}+y_{1}-\left(\bar{y}_{0}+\bar{y}_{1}\right) / \pi^{2} \neq 0$, problem (2) with $f\left(x, y, y^{\prime \prime}\right)=\pi^{4} y$ has no solution. And if $\bar{y}_{0}+\bar{y}_{1} \neq 0$, problem (2) with $f\left(x, y, y^{\prime \prime}\right)=-\pi^{2} y^{\prime \prime}$ has no solution.

The above two examples show that condition (3) is sharp.

3. The linear problem. Here we study the unique solvability of the linear problem (1). We prove the following general uniqueness and existence theorem which exhausts all nonresonant cases.

THEOREM 2. If $f(x) \neq j^{4} \pi^{4}, j=1,2, \ldots$, for $x \in[0,1]$ and $f$ is continuous on $[0,1]$, then for any chosen $y_{0}, y_{1}, \bar{y}_{0}, \bar{y}_{1}$, and arbitrary continuous function $g(x)$ over $[0,1]$ the boundary value problem (1) has a unique solution.

Note. In particular condition sup $f(x)<\pi^{4}$ meets the assumption of the theorem and hence part of the following argument in proving Theorem 2 provides a complete proof of the statement of Usmani [4].

PROOF OF THEOREM. The proof uses the Fredholm alternative and the Fourier expansions.

We can convert problem (1) into an integral equation over the space $C[0,1]$,

$$
y-T y=z,
$$

where

$$
\begin{gathered}
(T y)(x)=\int_{0}^{1} \int_{0}^{1} G(x, s) G(s, t) f(t) y(t) d t d s \\
z(x)=y_{0}+x\left(y_{1}-y_{0}\right)+\int_{0}^{1} G(x, s)\left[\bar{y}_{0}+s\left(\bar{y}_{1}-\bar{y}_{0}\right)+\int_{0}^{1} G(s, t) g(t) d t\right] d s
\end{gathered}
$$

and $G(x, s)$ is the Green's function introduced in $\S 2$. Now we have only to show that for any $z(x) \in C[0,1]$, equation (15) is uniquely solvable in the space $C[0,1]$. Since $T: C[0,1] \rightarrow C[0,1]$ is a linear compact map, by the well-known Fredholm alternative, we see that it will be enough to prove that the only solution of equation

$$
y-T y=0
$$

is the trivial solution $y=0$. We proceed as follows.

Convert equation (16) back into a boundary value problem

$$
\begin{aligned}
& d^{4} y / d x^{4}-f(x) y=0, \quad 0<x<1, \\
& y(0)=y(1)=y^{\prime \prime}(0)=y^{\prime \prime}(1)=0 .
\end{aligned}
$$


Assume $k \neq j^{4} \pi^{4}, j=1,2, \ldots$, and define a map $L: L^{2}[0,1] \rightarrow L^{2}[0,1]$ by $u=L v$, where $u$ and $v$ are related through

$$
\begin{aligned}
& d^{4} u / d x^{4}-k u=v(x), \quad 0<x<1, \\
& u(0)=u(1)=u^{\prime \prime}(0)=u^{\prime \prime}(1)=0 .
\end{aligned}
$$

Since $\{\sqrt{2} \sin (j \pi x)\}_{j=1,2, \ldots}$ is a complete orthonormal basis of $L^{2}[0,1]$, we have, in the space $L^{2}[0,1]$,

$$
u(x)=\sqrt{2} \sum_{j=1}^{\infty} a_{j} \sin (j \pi x), \quad v(x)=\sqrt{2} \sum_{j=1}^{\infty} b_{j} \sin (j \pi x)
$$

where

$$
a_{j}=b_{j} /\left(j^{4} \pi^{4}-k\right), \quad j=1,2, \ldots
$$

Hence by the Parseval equality we reach

$$
\begin{aligned}
\|u\|_{L}^{2} 2 & =\sum_{j=1}^{\infty}\left|a_{j}\right|^{2}=\sum_{j=1}^{\infty} \frac{\left|b_{j}\right|^{2}}{\left(j^{4} \pi^{4}-k\right)^{2}} \\
& \leq c \sum_{j=1}^{\infty}\left|b_{j}\right|^{2}=c\|v\|_{L^{2}}^{2},
\end{aligned}
$$

and

$$
\begin{aligned}
c & =c(k)=1 /\left(\pi^{4}-k\right)^{2}, \quad \text { if } k<\pi^{4}, \\
& =1 / \min \left\{\left(j^{4} \pi^{4}-k\right)^{2},\left([j+1]^{4} \pi^{4}-k\right)^{2}\right\}, \quad \text { if } k \in\left(j^{4} \pi^{4},[j+1]^{4} \pi^{4}\right) .
\end{aligned}
$$

Therefore it follows from (18) that

$$
\|L\|^{2} \leq c
$$

On the other hand, we can rewrite problem (17) as

$$
\begin{aligned}
& d^{4} y / d x^{4}-k y=(f(x)-k) y(x), \quad 0<x<1, \\
& y(0)=y(1)=y^{\prime \prime}(0)=y^{\prime \prime}(1)=0 .
\end{aligned}
$$

If we define a map $K: L^{2}[0,1] \rightarrow L^{2}[0,1]$ by

$$
(K u)(x)=L([f-k] u)(x), \quad u(x) \in L^{2}[0,1],
$$

then $K$ is a linear operator satisfying

$$
\|K u\|_{L^{2}}^{2} \leq\|L\|^{2} \sup (f(x)-k)^{2}\|u\|_{L^{2}}^{2} .
$$

Because $f(x) \neq j^{4} \pi^{4}, j=1,2, \ldots$, and by the connectedness of $f([0,1])$ we see that either

$$
\sup f(x)<\pi^{4}
$$

or for some integer $j>0$ there are constants $p$ and $q$ such that

$$
j^{4} \pi^{4}<p \leq \inf f(x) \leq \sup f(x) \leq q<(j+1)^{4} \pi^{4} .
$$

Using (19), (20) and (22) we easily conclude that we can choose $k$ according to $f$ satisfying (23) or (24) to make $\|K\|<1$. Consequently $K$ has only one fixed point 
in the space $L^{2}[0,1]$ which is $y=0$. This proves problem (21), hence problem (17) has only the trivial solution $y=0$.

The proof of theorem is complete.

Note. Since $\sin (j \pi x)$ is a nontrivial solution of problem (17) with $f(x)=j^{4} \pi^{4}$, the theorem obtained above exhausts all nonresonant cases and is sharp in a general sense.

\section{REFERENCES}

1. A. R. Aftabizadeh, Existence and uniqueness theorems for fourth-order boundary value problems, J. Math. Anal. Appl. 116 (1986), 415-426.

2. M. S. Berger, Nonlinearity and functional analysis, Academic Press, New York, 1977.

3. J. T. Schwartz, Nonlinear functional analysis, Gordon \& Breach, New York, 1969.

4. R. A. Usmani, A uniqueness theorem for a boundary value problem, Proc. Amer. Math. Soc. 77 (1979), 327-335.

5. Private communication with R. A. Usmani.

Department of Mathematics and Statistics, University of MassaChusetTs, AMHERST, MASSACHUSETTS 01003 